\title{
LETTERS
} \section{TO THE EDITOR}

\section{Synthesis and Structure (Z)-N-Aryl-2-hydroxy-4-oxo-4-phenylbut-2-enamides}

\author{
V. L. Gein ${ }^{a} *$, T. M. Zamaraeva ${ }^{a}$, E. V. Gorgopina ${ }^{a}$, \\ N. M. Igidov $^{a}$, O. V. Bobrovskaya ${ }^{a}$, and M. V. Dmitriev ${ }^{b}$ \\ ${ }^{a}$ Perm State Pharmaceutical Academy, ul. Polevaya 2, Perm, 614990 Russia \\ *e-mail: geinvl48@mail.ru \\ ${ }^{b}$ Perm State National Research University, Perm, Russia
}

Received November 2, 2017

\begin{abstract}
Reactions of 5-phenyl-2,3-dihydrofuran-2,3-dione with aromatic amines in anhydrous dioxane or methyl benzoylpyruvate with aromatic amines in the presence of sodium acetate in glacial acetic acid afforded (Z)- $N$-aryl-2-hydroxy-4-oxo-4-phenylbut-2-enamides.
\end{abstract}

Keywords: methyl benzoylpyruvate, 5-phenyl-2,3-dihydrofuran-2,3-dione, arylamines, aroylpyruvic acid amides

DOI: $10.1134 / \mathrm{S} 1070363218040321$

Aroylpyruvic acid amides are of obvious chemical interest. These polyfunctional reagents show high reactivity due to the presence of several reaction sites in their structure [1,2]. They can act as C-nucleophiles and carbonyl electrophiles in the reactions, being promising starting materials for the synthesis of a variety of acyclic and heterocyclic compounds [1-4].

The most studied method for the preparation of $\mathrm{N}$-substituted amides of aroylpyruvic acids is the ring opening of 5-aryl-2,3-dihydrofuran-2,3-diones under the action of $N$-nucleophiles in an inert solvent medium with an equimolar ratio of the reagents $[5,6]$. Because of a number of drawbacks in this method [7], it remains urgent to find optimal conditions for the synthesis of amides of aroylpyruvic acids, which due to widely varying pharmacophore groups in their structure can be used further in the preparation of previously unknown and inaccessible functional heterocyclic compounds.

A new simple method for the preparation of $N$ arylamides of aroylpyruvic acids reported in [8] includes the reaction of arylamines with methyl aroylpyruvates in acetic acid in the presence of anhydrous sodium acetate. Later, arylamides obtained have been modified with 4-aminobenzenesulfonylacetamide sodium (sodium sulfacetamide) [9], 4-aminobenzenesulfonylguanidine (sulgine) [10], streptocide, and norsulfazole fragments [11].

Scheme 1.

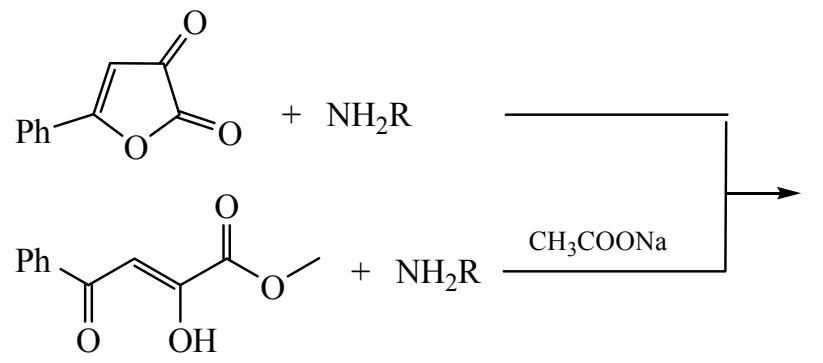<smiles>[R]NC(=O)C(=O)CC(=O)c1ccccc1</smiles>

$\mathrm{R}=\mathrm{C}_{6} \mathrm{H}_{5}$ (1), 4- $-\mathrm{BrC}_{6} \mathrm{H}_{4}$ (2), 4- $\mathrm{IC}_{6} \mathrm{H}_{4}$ (3), 3- $\mathrm{ClC}_{6} \mathrm{H}_{4}$ (4), 4- $\mathrm{C}_{2} \mathrm{H}_{5} \mathrm{OOCC}_{6} \mathrm{H}_{4}$ (5), 4- $\mathrm{CH}_{3} \mathrm{C}_{6} \mathrm{H}_{4}$ (6), 4- $\mathrm{CH}_{3} \mathrm{OC}_{6} \mathrm{H}_{4}$ (7), $4-\mathrm{NH}_{2} \mathrm{SO}_{2} \mathrm{C}_{6} \mathrm{H}_{4} \mathrm{CH}_{2} \mathrm{CH}_{2}$ (8). 
Continuing research in this direction, we apply this method for the synthesis of new $N$-arylamides of aroylpyruvic acids and studied their spatial structure. For the comparative study, (Z)- $N$-aryl-2-hydroxy-4oxo-4-phenylbut-2-enamides were prepared by reacting 5-phenyl-2,3-dihydrofuran-2,3-dione with anilines in an anhydrous dioxane.

To establish the possibility of using alkylamines in the synthesis of aroylpyruvic acid amides, we performed the reaction with 4-(2-aminoethyl) benzenesulfonamide by two aforementioned methods. However, we did not succeed in obtaining compound $\mathbf{8}$ according to method $b$.

Compounds 1-8 are yellow crystalline substances soluble in DMF, DMSO, soluble at heating in acetic acid, ethanol, and insoluble in water (Scheme 1).

In the ${ }^{1} \mathrm{H}$ NMR spectra of compounds $\mathbf{1 - 8}$, there were singlets of $\mathrm{COCH}_{2} \mathrm{CO}$ (4.53-4.64 ppm), $\mathrm{CH}=$ (7.02-7.17 ppm), CONH groups (8.99-10.78 pm), as well as the signals of aromatic fragments. In the spectrum of $\mathbf{8}$, the protons of both $\mathrm{CH}_{2}$ and $\mathrm{NH}_{2}$ groups were recorded in the regions of 2.86-3.51 and 7.32 ppm, respectively.

Crystal structure of compound $\mathbf{5}$ was studied by single-crystal X-ray diffraction method (see the figure). The crystals were obtained by slow crystallization from acetic acid. The molecule of $\mathbf{5}$ crystallizes in the centrosymmetric spatial group of the monoclinic crystal system. The molecule is practically planar. The electron density of the keto-enol fragment is strongly delocalized, which is expressed in equalizing the lengths of multiple and single $\mathrm{C}-\mathrm{C}$ and $\mathrm{C}-\mathrm{O}$ bonds (see Table). The position of the hydrogen atom of the enol hydroxy group was refined taking into account the disordering due to the existence of tautomeric equilibrium [the ratio of the populations of the $\mathrm{H}^{4}$ and $\mathrm{H}^{5}$ atoms is $0.59(7)$ to $0.41(7)$, respectively]. In addition to the intramolecular hydrogen bond in the keto-enol fragment the molecule also has an intramolecular hydrogen bond of non-classical type $\mathrm{C}^{6}-\mathrm{H}^{6} \cdots \mathrm{O}^{3}\left[\mathrm{H}^{6} \cdots \mathrm{O}^{3} 2.329, \mathrm{C}^{6} \cdots \mathrm{O}^{3} 2.927(4) \AA\right]$. The crystal packing is stabilized by van der Waals interactions and several shortened $\mathrm{C}-\mathrm{H} \cdots \mathrm{O}$ contacts.

According to the studies performed, the most effective and convenient method for the synthesis of (Z)- $N$-aryl-2-hydroxy-4-oxo-4-phenylbut-2-enamides containing arylamine fragment with an electronwithdrawing group is the reaction of benzoylpyruvic acid methyl ester with arylamines in glacial acetic acid

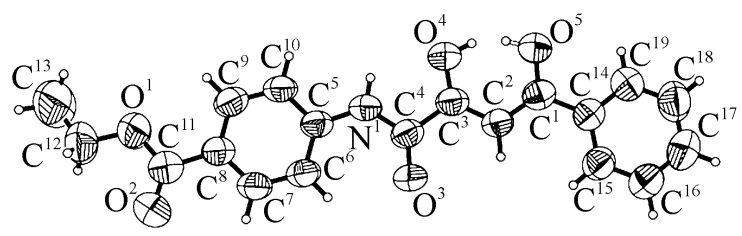

General view of the molecule of compound $\mathbf{5}$ in the crystal represented by thermal ellipsoids with $50 \%$ probability.

in the presence of anhydrous sodium acetate. When using an aromatic amine with an electron-donor substituent or an alkylamine for the preparation of benzoylpyruvic acid amides, it is more expedient to use the ring opening reaction of 5-aryl-2,3-dihydrofuran-2,3-dione in dioxane.

(Z)-2-Hydroxy-4-oxo- $N$,4-diphenylbut-2-enamide (1). $a$. To $1.0 \mathrm{~g}(0.0057 \mathrm{~mol})$ of 5-phenyl-2,3-dihydrofuran-2,3-dione in $20 \mathrm{~mL}$ of anhydrous dioxane was added $0.52 \mathrm{~mL}(0.0057 \mathrm{~mol})$ of aniline in $2 \mathrm{~mL}$ of anhydrous dioxane. Then the solvent was distilled off, the precipitate was washed with ethyl alcohol.

b. A mixture of $8.24 \mathrm{~g}(0.04 \mathrm{~mol})$ of methyl benzoylpyruvate and $3.28 \mathrm{~g}(0.04 \mathrm{~mol})$ of anhydrous sodium acetate in $10 \mathrm{~mL}$ of glacial acetic acid was added to $3.65 \mathrm{~mL}(0.04 \mathrm{~mol})$ of aniline dissolved in glacial acetic acid. The reaction mixture was refluxed for 20-30 min. The residue was filtered off and recrystallized from acetic acid. Yield $1.09 \mathrm{~g}(72 \%$, method $a), 6.74 \mathrm{~g}(63 \%$, method $b), \operatorname{mp} 90-92^{\circ} \mathrm{C} .{ }^{1} \mathrm{H}$ NMR spectrum (DMSO- $\left.d_{6}\right), \delta$, ppm: $4.63 \mathrm{~s}(2 \mathrm{H}$, $\left.\mathrm{COCH}_{2} \mathrm{CO}\right), 7.02 \mathrm{~s}(1 \mathrm{H}, \mathrm{CH}=), 7.12 \mathrm{t}(1 \mathrm{H}, \mathrm{ArH}, J=$ $7.5 \mathrm{~Hz}), 7.35 \mathrm{t}(2 \mathrm{H}, \mathrm{ArH}, J=7.5 \mathrm{~Hz}), 7.52 \mathrm{t}(1 \mathrm{H}, \mathrm{ArH}$, $J=6.9 \mathrm{~Hz}), 7.59 \mathrm{t}(2 \mathrm{H}, \mathrm{ArH}, J=6.9 \mathrm{~Hz}), 7.79 \mathrm{~d}(2 \mathrm{H}$, $\operatorname{ArH}, J=7.5 \mathrm{~Hz}), 7.98 \mathrm{~d}(2 \mathrm{H}, \mathrm{ArH}, J=6.9 \mathrm{~Hz}), 10.37$ s $(1 \mathrm{H}, \mathrm{CONH})$. Found, \%: C 70.98, 72.02; H 4.82, 4.99; $\mathrm{N}$ 5.12, 5.37. $\mathrm{C}_{16} \mathrm{H}_{13} \mathrm{NO}_{3}$. Calculated, \%: C 71.90; H 4.90; N 5.24.

(Z)-N-(4-Bromophenyl)-2-hydroxy-4-oxo-4-phenylbut-2-enamide (2). Yield $1.55 \mathrm{~g}$ (77\%, method $a$ ), $5.95 \mathrm{~g}(86 \%$, method $b)$, mp $148-150{ }^{\circ} \mathrm{C}$. ${ }^{1} \mathrm{H}$ NMR

Some bond lengths in the molecule of 5

\begin{tabular}{c|c|c|c}
\hline Bond & $d, \AA$ & Bond & $d, \AA$ \\
\hline $\mathrm{C}^{3}-\mathrm{C}^{4}$ & $1.515(4)$ & $\mathrm{C}^{2}=\mathrm{C}^{3}$ & $1.381(4)$ \\
$\mathrm{C}^{4}-\mathrm{N}^{1}$ & $1.349(4)$ & $\mathrm{C}^{2}-\mathrm{C}^{1}$ & $1.391(5)$ \\
$\mathrm{N}^{1}-\mathrm{C}^{5}$ & $1.402(4)$ & $\mathrm{C}^{1}=\mathrm{O}^{5}$ & $1.297(4)$ \\
$\mathrm{C}^{4}=\mathrm{O}^{3}$ & $1.211(4)$ & $\mathrm{C}^{1}-\mathrm{C}^{14}$ & $1.479(5)$ \\
$\mathrm{C}^{3}-\mathrm{O}^{4}$ & $1.267(4)$ & $\mathrm{C}^{8}-\mathrm{C}^{11}$ & $1.478(5)$ \\
\hline
\end{tabular}


spectrum (DMSO- $\left.d_{6}\right), \delta$, ppm: $4.62 \mathrm{~s}\left(2 \mathrm{H}, \mathrm{COCH}_{2} \mathrm{CO}\right)$, $7.17 \mathrm{~s}(1 \mathrm{H}, \mathrm{CH}=), 7.56 \mathrm{~d}(1 \mathrm{H}, \mathrm{ArH}, J=8.7 \mathrm{~Hz}), 7.61 \mathrm{~d}$ $(1 \mathrm{H}, \mathrm{ArH}, J=8.7 \mathrm{~Hz}), 7.68 \mathrm{t}(3 \mathrm{H}, \operatorname{ArH}, J=7.2 \mathrm{~Hz})$, $7.76 \mathrm{~d}(1 \mathrm{H}, \mathrm{ArH}, J=8.7 \mathrm{~Hz}), 7.81 \mathrm{~d}(1 \mathrm{H}, \operatorname{ArH}, J=$ $8.7 \mathrm{~Hz}), 7.99 \mathrm{~d}(1 \mathrm{H}, \mathrm{ArH}, J=7.2 \mathrm{~Hz}), 8.06 \mathrm{~d}(1 \mathrm{H}$, $\mathrm{ArH}, J=7.5 \mathrm{~Hz}), 10.59 \mathrm{~s}(1 \mathrm{H}, \mathrm{CONH})$. Found, \%: C 55.39, 55.62; H 3.39, 3.58; N 3.92, 4.17. $\mathrm{C}_{16} \mathrm{H}_{12} \mathrm{BrNO}_{3}$. Calculated, \%: C 55.51; H 3.49; N 4.05.

(Z)-N-(4-Iodophenyl)-2-hydroxy-4-oxo-4-phenylbut-2-enamide (3). Yield $1.85 \mathrm{~g}$ (83\%, method $a$ ), $7.3 \mathrm{~g}(93 \%$, method $b), \mathrm{mp} 134-136^{\circ} \mathrm{C} .{ }^{1} \mathrm{H}$ NMR spectrum (DMSO- $\left.d_{6}\right), \delta$, ppm: $4.61 \mathrm{~s}\left(2 \mathrm{H}, \mathrm{COCH}_{2} \mathrm{CO}\right)$, $7.16 \mathrm{~s}(1 \mathrm{H}, \mathrm{CH}=), 7.55-8.06 \mathrm{~m}(9 \mathrm{H}, \mathrm{ArH}), 10.55 \mathrm{~s}$ (1H, CONH). Found, \%: C 48.77, 49.01; H 2.98, 3.17; $\mathrm{N} 3.44,3.67 . \mathrm{C}_{16} \mathrm{H}_{12} \mathrm{INO}_{3}$. Calculated, \%: C 48.88; H $3.08 ; \mathrm{N} 3.56$.

(Z)-N-(3-Chlorophenyl)-2-hydroxy-4-oxo-4-phenylbut-2-enamide (4). Yield $4.5 \mathrm{~g}(75 \%$, method $b)$, mp $242-244^{\circ} \mathrm{C}$. ${ }^{1} \mathrm{H}$ NMR spectrum (DMSO- $d_{6}$ ), $\delta$, ppm: $4.63 \mathrm{~s}\left(2 \mathrm{H}, \mathrm{COCH}_{2} \mathrm{CO}\right), 7.15 \mathrm{~s}(1 \mathrm{H}, \mathrm{CH}=), 7.18-8.05$ $\mathrm{m}(9 \mathrm{H}, \mathrm{ArH}), 10.62 \mathrm{~s}(1 \mathrm{H}, \mathrm{CONH})$. Found, \%: C 63.59, 63.81; H 3.94, 4.10; N 4.51, 4.75. $\mathrm{C}_{16} \mathrm{H}_{12} \mathrm{ClNO}_{3}$. Calculated, \%: C 63.69; H 4.01; N 4.64.

(Z)-Ethyl 4-\{[2-hydroxy-4-oxo-4-phenylbut-2-enoyl]amino\}benzoate (5). Yield $10.95 \mathrm{~g}(81 \%$, method $b)$, mp $127-129^{\circ} \mathrm{C}(\mathrm{EtOH}) .{ }^{1} \mathrm{H}$ NMR spectrum (DMSO$\left.d_{6}\right), \delta$, ppm: $1.36 \mathrm{t}\left(3 \mathrm{H}, \mathrm{CH}_{3} \mathrm{CH}_{2} \mathrm{O}, J=6.9 \mathrm{~Hz}\right), 4.32 \mathrm{q}$ $\left(2 \mathrm{H}, \mathrm{CH}_{3} \mathrm{CH}_{2} \mathrm{O}, J=6.9 \mathrm{~Hz}\right), 4.64 \mathrm{~s}\left(2 \mathrm{H}, \mathrm{COCH}_{2} \mathrm{CO}\right)$, $7.16 \mathrm{~s}(1 \mathrm{H}, \mathrm{CH}=), 7.55-8.03 \mathrm{~m}(9 \mathrm{H}, \operatorname{ArH}), 10.78 \mathrm{~s}$ (1H, CONH). Found, \%: C 67.16, 67.38; H 4.95, 5.14; $\mathrm{N}$ 4.01, 4.24. $\mathrm{C}_{19} \mathrm{H}_{17} \mathrm{NO}_{5}$. Calculated, \%: C 67.25; $\mathrm{H}$ $5.05 ; \mathrm{N} 4.13$.

(Z)-2-Hydroxy-4-oxo-4-phenyl- $N$-(p-tolyl)but-2enamide (6). Yield $0.8 \mathrm{~g}(50 \%$, method $a)$, $\mathrm{mp}$ 92-94 ${ }^{\circ} \mathrm{C}(\mathrm{EtOH}) .{ }^{1} \mathrm{H}$ NMR spectrum (DMSO- $\left.d_{6}\right), \delta$, ppm: $2.29 \mathrm{~s}\left(3 \mathrm{H}, \mathrm{CH}_{3} \mathrm{C}_{6} \mathrm{H}_{4}\right), 4.61 \mathrm{~s}\left(2 \mathrm{H}, \mathrm{COCH}_{2} \mathrm{CO}\right)$, $7.16 \mathrm{~s}(1 \mathrm{H}, \mathrm{CH}=), 7.12-8.06 \mathrm{~m}(9 \mathrm{H}, \mathrm{ArH}), 10.34 \mathrm{~s}(1 \mathrm{H}$, CONH). Found, \%: C 72.47, 72.69; H 5.28, 5.45; N 4.86, 5.11. $\mathrm{C}_{17} \mathrm{H}_{15} \mathrm{NO}_{3}$. Calculated, \%: C 72.58; H 5.37; N 4.99 .

(Z)-2-Hydroxy- $N$-(4-methoxyphenyl)-4-oxo-4-

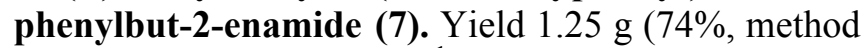
a), mp $110-112^{\circ} \mathrm{C}$ (EtOH). ${ }^{1} \mathrm{H}$ NMR spectrum (DMSO$\left.d_{6}\right), \delta$, ppm: $3.76 \mathrm{~s}\left(3 \mathrm{H}, \underline{\mathrm{CH}_{3}} \mathrm{OC}_{6} \mathrm{H}_{4}\right), 4.60 \mathrm{~s}(2 \mathrm{H}$, $\left.\mathrm{COCH}_{2} \mathrm{CO}\right), 7.17 \mathrm{~s}(1 \mathrm{H}, \overline{\mathrm{CH}}=), 6.92-8.03 \mathrm{~m}(9 \mathrm{H}$, $\mathrm{ArH}), 10.33 \mathrm{~s}(1 \mathrm{H}, \mathrm{CONH})$. Found, \%: C 68.56, 68.80; $\mathrm{H} 4.98,5.19 ; \mathrm{N} 4.59,4.84 . \mathrm{C}_{17} \mathrm{H}_{15} \mathrm{NO}_{4}$. Calculated, \%: C 68.68; H 5.09; N 4.71.
(2Z)-2-Hydroxy-4-oxo- $\mathrm{N}$-[2-(4-sulfamoylphenyl)ethyl]-4-phenylbut-2-enamide (8). Yield $1.75 \mathrm{~g}(82 \%$, method $a$ ), mp $165-167^{\circ} \mathrm{C}(\mathrm{EtOH}) .{ }^{1} \mathrm{H}$ NMR spectrum (DMSO- $\left.d_{6}\right), \delta$, ppm: $2.86 \mathrm{~m}\left(2 \mathrm{H}, \mathrm{C}^{2} \mathrm{H}_{2}\right), 2.95 \mathrm{~m}(1 \mathrm{H}$, $\left.\mathrm{C}^{1} \mathrm{H}_{\mathrm{B}}\right), 3.51 \mathrm{~m}\left(1 \mathrm{H}, \mathrm{C}^{1} \mathrm{H}_{\mathrm{A}}\right), 4.53 \mathrm{~s}\left(2 \mathrm{H}, \mathrm{COCH}_{2} \mathrm{CO}\right)$, $7.08 \mathrm{~s}(1 \mathrm{H}, \mathrm{CH}=), 7.32 \mathrm{~s}\left(1 \mathrm{H}, \mathrm{SO}_{2} \mathrm{NH}_{2}\right), 7.38-8.05 \mathrm{~m}$ $(9 \mathrm{H}, \mathrm{ArH}), 8.99 \mathrm{~s}(1 \mathrm{H}, \mathrm{CONH})$. Found, \%: C 57.63; H 4.80; N 7.55; $\mathrm{S}$ 8.65. $\mathrm{C}_{18} \mathrm{H}_{18} \mathrm{~N}_{2} \mathrm{O}_{5} \mathrm{~S}$. Calculated, \%: C 57.74; H 4.85; N 7.48; S 8.56.

${ }^{1} \mathrm{H}$ NMR spectra (DMSO- $d_{6}$ ) were registered on a Bruker 500 spectrometer $(500.13 \mathrm{MHz})$, internal reference TMS. Elemental analysis was performed using a Perkin Elmer 2400 analyzer. Melting points were determined on a Melting Point M-565 apparatus. $\mathrm{X}$-Ray diffraction analysis was performed on a Xcalibur Ruby diffractometer equipped with a CCD detector using the standard procedure $\left[\mathrm{Mo} K_{\alpha}\right.$-radiation, 295(2) K, $\omega$-scanning in $1^{\circ}$ increments] [12]. Empirical correction for absorption was performed using the SCALE3 ABSPACK algorithm [12]. The crystals are monoclinic, space group $P 2_{1} / c, a$ 12.779(4), $b$ 5.4942(17), c 24.676(9) $\AA, \beta$ 97.54(3) ${ }^{\circ}, V$ 1717.5(10) $\AA^{3}$, $Z$ 4. The structure was solved by the direct method using SHELX software [13] and refined by the fullmatrix least squares method with respect to $F^{2}$ in anisotropic approximation for all non-hydrogen atoms using SHELXL [14] and OLEX2 programs [15]. The positions of hydrogen atoms of the $\mathrm{NH}$ and $\mathrm{OH}$ groups were refined independently in isotropic approximation, and other atoms were refined using a rider model. For $\mathrm{O}-\mathrm{H}$ bond lengths, the values of $0.82 \AA$ were determined by the soft DFIX constraint. The final refinement parameters are as follows: $R_{1} 0.0659, w R_{2}$ 0.1711 [for 1558 reflections with $I>2 \sigma(I)$ ], $R_{1} 0.1781$, $w R_{2} 0.2402$ (for all 4031 independent reflections), $S$ 1.019. The X-ray diffraction data were deposited at the Cambridge Crystallographic Data Centre (CCDC 1567591).

\section{REFERENCES}

1. Andreichikov, Yu.S., Milyutin, A.V., Krylova, I.V., Saraeva, R.F., Dormidontova, E.V., Drovosekova, L.P., Nazmetdinov, F.Ya., and Kolla, V.E., Pharm. Chem. J., 1990, vol. 24, no. 7, p. 473. doi 10.1007/BF00764994

2. Milyutin, A.V., Doctoral (Pharm.) Dissertation, Perm, 1998.

3. Pulina, N.A., Mokin, P.A., Yushkov, V.V., Odegova, T.F., Tomilov, M.V., Yatsenko, K.V., and Zalesov, V.V., Pharm. Chem. J., 2008, vol. 42, no. 7, p. 389. doi 10.1007/s11094-008-0147-1 
4. Koz'minykh, E.N., Belyaev, A.O., Berezina, E.S., Koz'minykh, V.O., Makhmudov, R.R., and Odegova, T.F., Pharm. Chem. J., 2002, vol. 36, no. 12, p. 643. doi 10.1023/A:1023449426814

5. Andreichikov, Yu.S., Nalimova, Yu.A., Tendryakova, S.P., and Vilenchik, Ya.M., Zh. Org. Khim., 1978, vol. 14, no. 1, p. 160.

6. Kozlov, A.P., Sazhnev, S.S., Kozlova, G.A., and Andreichikov, Yu.S., Zh. Org. Khim., 1996, vol. 32, no. 10, p. 1573.

7. Tendryakova, S.P., Candidate Sci. (Chem.) Dissertation, Perm, 1981.

8. Gein, V.L., Bobrovskaya, O.V., and Gein, L.F., Russ. J. Org. Chem., 2014, vol. 50, no. 11, p. 1692. doi 10.1134/ S1070428014110268

9. Gein, V.L., Bobrovskaya, O.V., and Sitnikova, A.A.,
Russ. J. Gen. Chem., 2014, vol. 84, no. 4, p. 629. doi 10.1134/S1070363214040045

10. Gein, V.L., Bobrovskaya, O.V., Kovtonogova, I.V., and Novikova, V.V., Russ. J. Gen. Chem., 2015, vol. 85, no. 4, p. 833. doi 10.1134/S1070363215040106

11. Gein, V.L., Bobrovskaya, O.V., and Odegova, T.F., RF Patent 2624226, 2017.

12. CrysAlisPro, Agilent Technologies, Version 1.171.37.33 (release 27-03-2014 CrysAlis171.NET).

13. Sheldrick, G.M., Acta Crystallogr., 2008, vol. 64, p. 112. doi 10.1107/S0108767307043930

14. Sheldrick, G.M., Acta Crystallogr. (C), 2015, vol. 71, p. 3. doi 10.1107/S2053229614024218

15. Dolomanov, O.V., Bourhis, L.J., Gildea, R.J., Howard, J.A.K., and Puschmann, H., J. Appl. Crystallogr., 2009, vol. 42, p. 339. doi 10.1107/S0021889808042726 